Pacific

Journal of

Mathematics

SOME EISENSTEIN SERIES IDENTITIES RELATED TO MODULAR EQUATIONS OF THE SEVENTH ORDER

ZHI-GuO LIU

Volume 209 No. 1

March 2003 


\title{
SOME EISENSTEIN SERIES IDENTITIES RELATED TO MODULAR EQUATIONS OF THE SEVENTH ORDER
}

\author{
ZHI-GUO LIU \\ Dedicated to my friend Richard Lewis
}

In this paper we will use one well-known modular equation of seventh order, one theta function identity of S. McCullough and L.-C. Shen, 1994, and the complex variable theory of elliptic functions to prove some new septic identities for theta functions. Then we use these identities to provide new proofs of some Eisenstein series identities in Ramanujan's notebooks or "lost" notebook. We also derive a new identity for Eisenstein series and some curious trigonometric identities.

\section{Introduction.}

Suppose throughout that $q=\exp (2 \pi i \tau)$, where $\tau$ has positive imaginary part, and set

$$
(z ; q)_{\infty}=\prod_{n=0}^{\infty}\left(1-z q^{n}\right) .
$$

The Dedekind eta-function is defined by

$$
\eta(\tau)=q^{\frac{1}{24}}(q ; q)_{\infty}=e^{\frac{\pi i \tau}{12}} \prod_{n=1}^{\infty}\left(1-e^{2 \pi i n \tau}\right) .
$$

For brevity, we define

$$
h(\tau)=\frac{\eta^{4}(7 \tau)}{\eta^{4}(\tau)}, \quad k(\tau)=\frac{\eta^{7}(\tau)}{\eta(7 \tau)}, \quad \text { and } \quad \rho(\tau)=7 \frac{\eta(49 \tau)}{\eta(\tau)} .
$$

Throughout this article we will use $\left(\frac{n}{7}\right)$ to denote the Legendre symbol. The Eisenstein series $T(\tau), L(\tau), M(\tau)$, and $N(\tau)$ are defined by

$$
\begin{aligned}
& T(\tau)=1+2 \sum_{n=1}^{\infty}\left(\frac{n}{7}\right) \frac{q^{n}}{1-q^{n}}=1+2 \sum_{n=1}^{\infty}\left(\frac{n}{7}\right) \frac{e^{2 \pi i n \tau}}{1-e^{2 \pi i n \tau}}, \\
& L(\tau)=1-24 \sum_{n=1}^{\infty} \frac{n q^{n}}{1-q^{n}}=1-24 \sum_{n=1}^{\infty} \frac{n e^{2 \pi i n \tau}}{1-e^{2 \pi i n \tau}},
\end{aligned}
$$




$$
M(\tau)=1+240 \sum_{n=1}^{\infty} \frac{n^{3} q^{n}}{1-q^{n}}=1+240 \sum_{n=1}^{\infty} \frac{n^{3} e^{2 \pi i n \tau}}{1-e^{2 \pi i n \tau}},
$$

and

$$
N(\tau)=1-504 \sum_{n=1}^{\infty} \frac{n^{5} q^{n}}{1-q^{n}}=1-504 \sum_{n=1}^{\infty} \frac{n^{5} e^{2 \pi i n \tau}}{1-e^{2 \pi i n \tau}}
$$

In his lost notebook [17, p, 53], S. Ramanujan recorded without proofs formulas for $T(r \tau), L(r \tau), M(r \tau)$, and $N(r \tau)$, for certain positive integers $r$, as sums of quotients of Dedekind eta-functions. These particular quotients (called Hauptmoduls) frequently arise in the theory and applications of modular forms and elliptic functions. In particular, Ramanujan claimed that:

Theorem 1. Let $k(\tau), h(\tau), M(\tau)$ and $N(\tau)$ defined by (1.3), (1.6), and (1.7), respectively. Then we have

$$
\begin{gathered}
M(\tau)=k(\tau)^{4 / 3}\left(1+245 h(\tau)+2401 h^{2}(\tau)\right) \\
\cdot\left(1+13 h(\tau)+49 h^{2}(\tau)\right)^{1 / 3} \\
M(7 \tau)=k(\tau)^{4 / 3}\left(1+5 h(\tau)+h^{2}(\tau)\right)\left(1+13 h(\tau)+49 h^{2}(\tau)\right)^{1 / 3} \\
N(\tau)=k(\tau)^{2}\left(1-7^{2}(5+2 \sqrt{7}) h(\tau)-7^{3}(21+8 \sqrt{7}) h^{2}(\tau)\right) \\
\cdot\left(1-7^{2}(5-2 \sqrt{7}) h(\tau)-7^{3}(21-8 \sqrt{7}) h^{2}(\tau)\right),
\end{gathered}
$$

and

$$
\begin{array}{r}
N(7 \tau)=k(\tau)^{2}\left(1+(7+2 \sqrt{7}) h(\tau)+(21+8 \sqrt{7}) h^{2}(\tau)\right) \\
\cdot\left(1+(7-2 \sqrt{7}) h(\tau)+(21-8 \sqrt{7}) h^{2}(\tau)\right) .
\end{array}
$$

These identities reveal deep connections between Eisenstein series and Dedekind eta-functions. The first published proofs of (1.8)-(1.11) are due to S. Raghavan and S.S. Rangachari [16], who used the theory of modular forms with which Ramanujan was unfamiliar. These proofs give a uniform explanation of the existence of these identities but do not provide any insight into how Ramanujan discovered the identities. These proofs are essentially verifications. It is desirable to find more natural proofs of the aforementioned identities without employing the theory of modular forms. B.C. Berndt, H.H. Chan, J. Sohn, and S.H. Son [3] recently found proofs of (1.8)-(1.11) based entirely on results found in Ramanujan's notebooks [18]. In fact, their proofs depend upon some modular equations of the seventh order of Ramanujan. 
In the present paper, we present a quite different approach. Our main tools are the following three Lemmas:

Lemma 2. The sum of all the residues of an elliptic function at the poles inside a period-parallelogram is zero.

Lemma 3. Let $\theta_{1}(z \mid q)$ be Jacobi theta function defined by (2.1) below. Then:

$$
\begin{aligned}
& (q ; q)_{\infty} \frac{\theta_{1}(2 z \mid q)}{\theta_{1}(z \mid q)}=2 \sum_{n=-\infty}^{\infty}(-1)^{n} q^{\frac{1}{2}\left(3 n^{2}+n\right)} \cos (6 n+1) z \\
& \frac{\theta_{1}^{\prime}}{\theta_{1}}(x \mid q)+\frac{\theta_{1}^{\prime}}{\theta_{1}}(y \mid q)+\frac{\theta_{1}^{\prime}}{\theta_{1}}(z \mid q)-\frac{\theta_{1}^{\prime}}{\theta_{1}}(x+y+z \mid q) \\
& =\theta_{1}^{\prime}(0 \mid q) \frac{\theta_{1}(x+y \mid q) \theta_{1}(y+z \mid q) \theta_{1}(z+x \mid q)}{\theta_{1}(x \mid q) \theta_{1}(y \mid q) \theta_{1}(z \mid q) \theta_{1}(x+y+z \mid q)} .
\end{aligned}
$$

Lemma 4. Let $h(\tau)$ and $\rho(\tau)$ be defined by (1.3). Then

$$
\begin{gathered}
7 \rho^{3}(\tau)+35 \rho^{2}(\tau)+49 \rho(\tau)+\left(\rho^{2}(\tau)+7 \rho(\tau)+7\right) \\
\cdot \sqrt{4 \rho^{3}(\tau)+21 \rho^{2}(\tau)+28 \rho(\tau)}=98 h(\tau) .
\end{gathered}
$$

Lemma 2 is a fundamental theorem of elliptic functions and can be found in $[\mathbf{5}$, p. 22]. Recently, in $[\mathbf{9}, \mathbf{1 0}, \mathbf{1 1}, \mathbf{1 2}, \mathbf{1 3}]$, we have used Lemma 2 to set up many important theta function identities. Identity (1.12) is the well-known quintuple identity $[\mathbf{6}, \mathbf{7}, \mathbf{8}, \mathbf{2 1}]$. For an interesting account of this identity, one can consult $[\mathbf{2}$, p. 83]. Identity (1.13) was derived by S. McCullough and L.-C. Shen in their remarkable paper [14], in which they used the properties of theta functions to study the Sezgö kernel of an annulus. Identity (1.14) is $[\mathbf{2 2}$, p. 117, Equation (4.5)]. It plays a pivotal role in the study of the modular equations of degree 7 .

It should be emphasized that our method is constructive and can be used to derive theta function identities and Eisenstein series identities, rather than just to verify previously derived identities. This method provides deeper insight into the theory of theta function identities and Eisenstein series identities.

In this paper we will also prove the following identities:

Theorem 5. Let $k(\tau), h(\tau)$, and $T(\tau)$ be defined by (1.3) and (1.4), respectively. Then we have

$$
\begin{aligned}
& 8-7 \sum_{n=1}^{\infty}\left(\frac{n}{7}\right) \frac{n^{2} q^{n}}{1-q^{n}}=k(\tau)(8+49 h(\tau)), \\
& T(\tau)=k(\tau)^{1 / 3}\left(1+13 h(\tau)+49 h^{2}(\tau)\right)^{1 / 3},
\end{aligned}
$$




$$
\begin{aligned}
A(\tau): & =1+4 \sum_{n=1}^{\infty} \frac{n q^{n}}{1-q^{n}}-28 \sum_{n=1}^{\infty} \frac{n q^{7 n}}{1-q^{7 n}} \\
& =T^{2}(\tau)=k(\tau)^{2 / 3}\left(1+13 h(\tau)+49 h^{2}(\tau)\right)^{2 / 3}
\end{aligned}
$$

and

$$
\begin{aligned}
& 16+\sum_{n=1}^{\infty}\left(\frac{n}{7}\right) \frac{n^{4} q^{n}}{1-q^{n}}= k(\tau)^{5 / 3}(16+49 h(\tau)) \\
& \cdot\left(1+13 h(\tau)+49 h^{2}(\tau)\right)^{2 / 3} .
\end{aligned}
$$

Equation (1.15) can also be found in $[\mathbf{1 7}$, p. 53] and the first published proof of (1.15) are due to S. Raghavan [15], who used the theory of modular forms. Equations (1.16) and (1.17) are contained in Entry 5 (i) of Chapter 21 of Ramanujan's second notebook [18]. In [2, p, 467-473], B.C. Berndt has given proofs of (1.8) and (1.9) by using some modular equations of the seventh order. Many wonderful applications of (1.16) have been given in [10]. To the author's best knowledge (1.18) is a new identity.

In the course of our investigations, we obtain the following intriguing identities of theta functions:

Theorem 6. If $k(\tau), h(\tau)$ and $\rho(\tau)$ are defined by (1.3). Then we have

$$
\frac{\theta_{1}\left(\frac{2 \pi}{7} \mid q\right)}{\theta_{1}\left(\frac{\pi}{7} \mid q\right)}-\frac{\theta_{1}\left(\frac{3 \pi}{7} \mid q\right)}{\theta_{1}\left(\frac{2 \pi}{7} \mid q\right)}+\frac{\theta_{1}\left(\frac{\pi}{7} \mid q\right)}{\theta_{1}\left(\frac{3 \pi}{7} \mid q\right)}=1+\rho(\tau)
$$

$$
\begin{aligned}
& \frac{\theta_{1}\left(\frac{\pi}{7} \mid q\right)}{\theta_{1}\left(\frac{2 \pi}{7} \mid q\right)}-\frac{\theta_{1}\left(\frac{2 \pi}{7} \mid q\right)}{\theta_{1}\left(\frac{3 \pi}{7} \mid q\right)}+\frac{\theta_{1}\left(\frac{3 \pi}{7} \mid q\right)}{\theta_{1}\left(\frac{\pi}{7} \mid q\right)} \\
& =\frac{1}{2}(3 \rho(\tau)+4)+\frac{1}{2} \sqrt{4 \rho^{3}(\tau)+21 \rho^{2}(\tau)+28 \rho(\tau)}
\end{aligned}
$$

$$
\frac{\theta_{1}^{2}\left(\frac{\pi}{7} \mid q\right)}{\theta_{1}\left(\frac{3 \pi}{7} \mid q\right)}-\frac{\theta_{1}^{2}\left(\frac{2 \pi}{7} \mid q\right)}{\theta_{1}\left(\frac{\pi}{7} \mid q\right)}+\frac{\theta_{1}^{2}\left(\frac{3 \pi}{7} \mid q\right)}{\theta_{1}\left(\frac{2 \pi}{7} \mid q\right)}=0
$$

$$
\frac{\theta_{1}\left(\frac{2 \pi}{7} \mid q\right)}{\theta_{1}^{4}\left(\frac{\pi}{7} \mid q\right)}-\frac{\theta_{1}\left(\frac{\pi}{7} \mid q\right)}{\theta_{1}^{4}\left(\frac{3 \pi}{7} \mid q\right)}+\frac{\theta_{1}\left(\frac{3 \pi}{7} \mid q\right)}{\theta_{1}^{4}\left(\frac{2 \pi}{7} \mid q\right)}=\frac{1}{\sqrt{7}} \eta^{-2}(\tau) \eta^{-1}(7 \tau)(8+49 h(\tau))
$$

$$
\frac{\theta_{1}^{4}\left(\frac{3 \pi}{7} \mid q\right)}{\theta_{1}\left(\frac{\pi}{7} \mid q\right)}-\frac{\theta_{1}^{4}\left(\frac{\pi}{7} \mid q\right)}{\theta_{1}\left(\frac{2 \pi}{7} \mid q\right)}-\frac{\theta_{1}^{4}\left(\frac{2 \pi}{7} \mid q\right)}{\theta_{1}\left(\frac{3 \pi}{7} \mid q\right)}=\sqrt{7} \eta^{2}(\tau) \eta(7 \tau)(5+49 h(\tau)),
$$

$$
\frac{\theta_{1}^{7}\left(\frac{2 \pi}{7} \mid q\right)}{\theta_{1}^{7}\left(\frac{\pi}{7} \mid q\right)}-\frac{\theta_{1}^{7}\left(\frac{3 \pi}{7} \mid q\right)}{\theta_{1}^{7}\left(\frac{2 \pi}{7} \mid q\right)}+\frac{\theta_{1}^{7}\left(\frac{\pi}{7} \mid q\right)}{\theta_{1}^{7}\left(\frac{3 \pi}{7} \mid q\right)}=57+2 \times 7^{3} h(\tau)+7^{4} h^{2}(\tau)
$$




$$
\begin{aligned}
& \frac{\theta_{1}^{7}\left(\frac{\pi}{7} \mid q\right)}{\theta_{1}^{7}\left(\frac{2 \pi}{7} \mid q\right)}-\frac{\theta_{1}^{7}\left(\frac{2 \pi}{7} \mid q\right)}{\theta_{1}^{7}\left(\frac{3 \pi}{7} \mid q\right)}+\frac{\theta_{1}^{7}\left(\frac{3 \pi}{7} \mid q\right)}{\theta_{1}^{7}\left(\frac{\pi}{7} \mid q\right)} \\
& =289+18 \times 7^{3} h(\tau)+19 \times 7^{4} h^{2}(\tau)+7^{6} h^{3}(\tau),
\end{aligned}
$$

$$
\begin{aligned}
& \frac{\theta_{1}^{3}\left(\frac{3 \pi}{7} \mid q\right)}{\theta_{1}^{6}\left(\frac{\pi}{7} \mid q\right)}-\frac{\theta_{1}^{3}\left(\frac{\pi}{7} \mid q\right)}{\theta_{1}^{6}\left(\frac{2 \pi}{7} \mid q\right)}+\frac{\theta_{1}^{3}\left(\frac{2 \pi}{7} \mid q\right)}{\theta_{1}^{6}\left(\frac{3 \pi}{7} \mid q\right)} \\
& =\frac{1}{\sqrt{7}} \eta^{-2}(\tau) \eta^{-1}(7 \tau)\left(46+637 h(\tau)+49^{2} h^{2}(\tau)\right),
\end{aligned}
$$

$$
\begin{aligned}
& \left(\frac{\theta_{1}\left(\frac{3 \pi}{7} \mid q\right)}{\theta_{1}^{2}\left(\frac{\pi}{7} \mid q\right)}-\frac{\theta_{1}\left(\frac{\pi}{7} \mid q\right)}{\theta_{1}^{2}\left(\frac{2 \pi}{7} \mid q\right)}+\frac{\theta_{1}\left(\frac{2 \pi}{7} \mid q\right)}{\theta_{1}^{2}\left(\frac{3 \pi}{7} \mid q\right)}\right)^{3} \\
& =7 \sqrt{7} \eta^{-2}(\tau) \eta^{-1}(7 \tau)\left(1+13 h(\tau)+49 h^{2}(\tau)\right)
\end{aligned}
$$

and

$$
\begin{gathered}
\theta_{1}^{-7}\left(\frac{\pi}{7} \mid q\right)-\theta_{1}^{-7}\left(\frac{2 \pi}{7} \mid q\right)-\theta_{1}^{-7}\left(\frac{3 \pi}{7} \mid q\right) \\
=\sqrt{7} \eta^{-14}(\tau) \eta^{-7}(7 \tau)(1+7 h(\tau)) \\
\cdot\left(1+13 h(\tau)+49 h^{2}(\tau)\right)^{1 / 3} .
\end{gathered}
$$

Using the product representation of $\theta_{1}(z \mid q)$ given by (2.2) and letting $q \rightarrow 0$ in (1.19)-(1.28), we readily find the following curious trigonometric identities:

Corollary 7. We have:

$$
\begin{aligned}
& \frac{\sin (2 \pi / 7)}{\sin (\pi / 7)}-\frac{\sin (3 \pi / 7)}{\sin (2 \pi / 7)}+\frac{\sin (\pi / 7)}{\sin (3 \pi / 7)}=1, \\
& \frac{\sin (\pi / 7)}{\sin (2 \pi / 7)}-\frac{\sin (2 \pi / 7)}{\sin (3 \pi / 7)}+\frac{\sin (3 \pi / 7)}{\sin (\pi / 7)}=2,
\end{aligned}
$$$$
\frac{\sin ^{2}(\pi / 7)}{\sin (3 \pi / 7)}-\frac{\sin ^{2}(2 \pi / 7)}{\sin (\pi / 7)}+\frac{\sin ^{2}(3 \pi / 7)}{\sin (2 \pi / 7)}=0,
$$$$
\frac{\sin (2 \pi / 7)}{\sin ^{4}(\pi / 7)}-\frac{\sin (\pi / 7)}{\sin ^{4}(3 \pi / 7)}+\frac{\sin (3 \pi / 7)}{\sin ^{4}(2 \pi / 7)}=\frac{64}{7} \sqrt{7},
$$$$
\frac{\sin ^{4}(3 \pi / 7)}{\sin (\pi / 7)}-\frac{\sin ^{4}(\pi / 7)}{\sin (2 \pi / 7)}-\frac{\sin ^{4}(2 \pi / 7)}{\sin (3 \pi / 7)}=\frac{5}{8} \sqrt{7},
$$$$
\frac{\sin ^{7}(2 \pi / 7)}{\sin ^{7}(\pi / 7)}-\frac{\sin ^{7}(3 \pi / 7)}{\sin ^{7}(2 \pi / 7)}+\frac{\sin ^{7}(\pi / 7)}{\sin ^{7}(3 \pi / 7)}=57,
$$$$
\frac{\sin ^{7}(\pi / 7)}{\sin ^{7}(2 \pi / 7)}-\frac{\sin ^{7}(2 \pi / 7)}{\sin ^{7}(3 \pi / 7)}+\frac{\sin ^{7}(3 \pi / 7)}{\sin ^{7}(\pi / 7)}=289,
$$ 


$$
\begin{aligned}
\frac{\sin ^{3}(3 \pi / 7)}{\sin ^{6}(\pi / 7)}-\frac{\sin ^{3}(\pi / 7)}{\sin ^{6}(2 \pi / 7)}+\frac{\sin ^{3}(2 \pi / 7)}{\sin ^{6}(3 \pi / 7)} & =\frac{368}{\sqrt{7}} \\
\frac{\sin (2 \pi / 7)}{\sin ^{2}(3 \pi / 7)}-\frac{\sin (\pi / 7)}{\sin ^{2}(2 \pi / 7)}+\frac{\sin (3 \pi / 7)}{\sin ^{2}(\pi / 7)} & =2 \sqrt{7} \\
\csc ^{7}\left(\frac{\pi}{7}\right)-\csc ^{7}\left(\frac{2 \pi}{7}\right)-\csc ^{7}\left(\frac{3 \pi}{7}\right) & =2^{7} \sqrt{7}
\end{aligned}
$$

Equations (1.31) and (1.37) have been found by Berndt and Zhang [4].

The rest of the article is organized as follows: In Section 2 we introduce some basic facts about theta function $\theta_{1}(z \mid q)$. In Section 3 we prove (1.19) using the quintuple product identity. Section 4 is devoted to the proofs of (1.20) and (1.21). In Section 5 we derive (1.22) and (1.23). Sections 6 and 7 are devoted to the proofs of (1.24)-(1.28). In Section 8 we prove (1.15), (1.16), and (1.17). In Sections 9 and 10 we derive (1.8)-(1.11). Lastly, in Section 11 we prove (1.18).

\section{Some basic facts about $\theta_{1}(z \mid \tau)$.}

We begin with the definition of the classical theta function $\theta_{1}(z \mid q)[\mathbf{2 3}, \mathrm{p}$. 464]

$$
\begin{aligned}
\theta_{1}(z \mid q) & =-i q^{\frac{1}{8}} \sum_{n=-\infty}^{\infty}(-1)^{n} q^{\frac{1}{2} n(n+1)} e^{(2 n+1) i z} \\
& =2 q^{\frac{1}{8}} \sum_{n=0}^{\infty}(-1)^{n} q^{\frac{1}{2} n(n+1)} \sin (2 n+1) z .
\end{aligned}
$$

Using the Jacobi triple product formula we have [23, p. 470]

$$
\theta_{1}(z \mid q)=2 q^{\frac{1}{8}}(\sin z)(q ; q)_{\infty}\left(q e^{2 i z} ; q\right)_{\infty}\left(q e^{-2 i z} ; q\right)_{\infty} .
$$

Differentiating the above equation with respect to $z$ and then putting $z=0$ we find that

$$
\theta_{1}^{\prime}(0 \mid q)=2 q^{\frac{1}{8}}(q ; q)_{\infty}^{3}=2 \eta^{3}(\tau),
$$

where and throughout this paper the prime means the partial derivative with respective to $z$.

From the definition of $\theta_{1}(z \mid q)$, the functional equations

$$
\theta_{1}(z+\pi \mid q)=-\theta_{1}(z \mid q), \quad \theta_{1}(z+\pi \tau \mid q)=-q^{-1 / 2} e^{-2 \pi i z} \theta_{1}(z \mid q)
$$

can be easily verified. Differentiating the above equations with respect to $z$, and then setting $z=0$, we find that

$$
\theta_{1}^{\prime}(\pi \mid q)=-\theta_{1}^{\prime}(0 \mid q), \quad \theta_{1}^{\prime}(\pi \tau \mid q)=-q^{-1 / 2} \theta_{1}^{\prime}(0 \mid q) .
$$


Taking $z=\frac{\pi}{7}, \frac{2 \pi}{7}$, and $\frac{3 \pi}{7}$, respectively in (2.2) and then multiplying the three resulting equations together we find that

$$
\theta_{1}\left(\frac{\pi}{7} \mid q\right) \theta_{1}\left(\frac{2 \pi}{7} \mid q\right) \theta_{1}\left(\frac{3 \pi}{7} \mid q\right)=\sqrt{7} q^{\frac{3}{8}}(q ; q)_{\infty}^{2}\left(q^{7} ; q^{7}\right)_{\infty}=\sqrt{7} \eta^{2}(\tau) \eta(7 \tau) .
$$

The Fourier series expansion for the logarithmic derivatives of $\theta_{1}(z \mid q)[\mathbf{2 3}$, p. 489] is

$$
\frac{\theta_{1}^{\prime}}{\theta_{1}}(z \mid q)=\cot z+4 \sum_{n=1}^{\infty} \frac{q^{n}}{1-q^{n}} \sin 2 n z
$$

Substituting

$$
\cot z=\frac{1}{z}-\frac{z}{3}-\frac{z^{3}}{45}-\frac{2 z^{5}}{945}-\frac{z^{7}}{4725}+\cdots
$$

and

$$
\sin z=z-\frac{1}{3 !} z^{3}+\frac{1}{5 !} z^{5}-\frac{1}{7 !} z^{7}+\cdots
$$

into (2.7) gives

$$
\begin{aligned}
\frac{\theta_{1}^{\prime}}{\theta_{1}}(z \mid q)= & \frac{1}{z}-\frac{1}{3} L(\tau) z-\frac{1}{45} M(\tau) z^{3}-\frac{2}{945} N(\tau) z^{5} \\
& -\frac{1}{4725}\left(1+480 \sum_{n=1}^{\infty} \frac{n^{7} q^{n}}{1-q^{n}}\right) z^{7}+\cdots
\end{aligned}
$$

By the infinite products expansion for $\theta_{1}(z \mid q)$ and direct computation, we find that

$$
\theta_{1}\left(7 z \mid q^{7}\right)=-\frac{\left(q^{7} ; q^{7}\right)_{\infty}}{(q ; q)_{\infty}^{7}} \theta_{1}(z \mid q) \prod_{r=1}^{3} \theta_{1}\left(z-\frac{r \pi}{7} \mid q\right) \theta_{1}\left(z+\frac{r \pi}{7} \mid q\right) .
$$

We now take the logarithmic derivative of this equation and obtain

$$
\sum_{r=1}^{3} \frac{\theta_{1}^{\prime}}{\theta_{1}}\left(z-\frac{r \pi}{7} \mid q\right)+\sum_{r=1}^{3} \frac{\theta_{1}^{\prime}}{\theta_{1}}\left(z+\frac{r \pi}{7} \mid q\right)=7 \frac{\theta_{1}^{\prime}}{\theta_{1}}\left(7 z \mid q^{7}\right)-\frac{\theta_{1}^{\prime}}{\theta_{1}}(z \mid \tau) .
$$

Using (2.10) on the right-hand side of (2.12) yields

$$
\begin{aligned}
& \sum_{r=1}^{3} \frac{\theta_{1}^{\prime}}{\theta_{1}}\left(z-\frac{r \pi}{7} \mid q\right)+\sum_{r=1}^{3} \frac{\theta_{1}^{\prime}}{\theta_{1}}\left(z+\frac{r \pi}{7} \mid q\right) \\
& =\frac{1}{3}\left(L(\tau)-7^{2} L(7 \tau)\right) z+\frac{1}{45}\left(M(\tau)-7^{4} M(7 \tau)\right) z^{3}+O\left(z^{5}\right) .
\end{aligned}
$$


Differentiating with repect to $z$ and then setting $z=0$ gives

$$
\left(\frac{\theta_{1}^{\prime}}{\theta_{1}}\right)^{\prime}\left(\frac{\pi}{7} \mid q\right)+\left(\frac{\theta_{1}^{\prime}}{\theta_{1}}\right)^{\prime}\left(\frac{2 \pi}{7} \mid q\right)+\left(\frac{\theta_{1}^{\prime}}{\theta_{1}}\right)^{\prime}\left(\frac{3 \pi}{7} \mid q\right)=\frac{1}{6}\left(L(\tau)-7^{2} L(7 \tau)\right) .
$$

Differentiating $(2.13)$ with repect to $z$, three times, and then setting $z=0$ we obtain

$$
\begin{aligned}
& \left(\frac{\theta_{1}^{\prime}}{\theta_{1}}\right)^{\prime \prime \prime}\left(\frac{\pi}{7} \mid q\right)+\left(\frac{\theta_{1}^{\prime}}{\theta_{1}}\right)^{\prime \prime \prime}\left(\frac{2 \pi}{7} \mid q\right)+\left(\frac{\theta_{1}^{\prime}}{\theta_{1}}\right)^{\prime \prime \prime}\left(\frac{3 \pi}{7} \mid q\right) \\
& =\frac{1}{15}\left(M(\tau)-7^{4} M(7 \tau)\right) .
\end{aligned}
$$

\section{The proof of (1.19).}

We recall the quintuple product identity (see Lemma 3)

$$
(q ; q)_{\infty} \frac{\theta_{1}(2 z \mid q)}{\theta_{1}(z \mid q)}=2 \sum_{n=-\infty}^{\infty}(-1)^{n} q^{\frac{1}{2}\left(3 n^{2}+n\right)} \cos (6 n+1) z .
$$

When $z=0,(3.1)$ reduces to the Euler identity

$$
(q ; q)_{\infty}=\sum_{n=-\infty}^{\infty}(-1)^{n} q^{\frac{1}{2}\left(3 n^{2}+n\right)} .
$$

Denote

$$
s(n):=\cos \frac{(6 n+1) \pi}{7}-\cos \frac{2(6 n+1) \pi}{7}+\cos \frac{3(6 n+1) \pi}{7} .
$$

By taking $z=\frac{\pi}{7}, z=-\frac{2 \pi}{7}$, and $z=\frac{3 \pi}{7}$, respectively, in (3.1) and then adding the resulting equations we obtain

$$
\begin{aligned}
& (q ; q)_{\infty}\left\{\frac{\theta_{1}\left(\frac{2 \pi}{7} \mid q\right)}{\theta_{1}\left(\frac{\pi}{7} \mid q\right)}-\frac{\theta_{1}\left(\frac{3 \pi}{7} \mid q\right)}{\theta_{1}\left(\frac{2 \pi}{7} \mid q\right)}+\frac{\theta_{1}\left(\frac{\pi}{7} \mid q\right)}{\theta_{1}\left(\frac{3 \pi}{7} \mid q\right)}\right\} \\
& =2 \sum_{n=-\infty}^{\infty}(-1)^{n} q^{\frac{1}{2}\left(3 n^{2}+n\right)} s(n) .
\end{aligned}
$$

From the following easily verified elementary trigonometric facts:

$$
s(n)=\left\{\begin{array}{lll}
-3, & n \equiv 1 & (\bmod 7) \\
\frac{1}{2}, & n \neq 1 & (\bmod 7),
\end{array}\right.
$$


we have the evaluation

$$
\begin{aligned}
& 2 \sum_{n=-\infty}^{\infty}(-1)^{n} q^{\frac{1}{2}\left(3 n^{2}+n\right)} s(n) \\
& =2 \sum_{\substack{n=-\infty \\
n \neq 1}}^{\infty}(-1)^{n} q^{\frac{1}{2}\left(3 n^{2}+n\right)} s(n)+2 \sum_{\substack{n=-\infty \\
n \equiv 1}}^{\infty}(-1)^{n} q^{\frac{1}{2}\left(3 n^{2}+n\right)} s(n) \\
& =\sum_{\substack{n \neq 1 \\
n=-\infty}}^{\infty}(-1)^{n} q^{\frac{1}{2}\left(3 n^{2}+n\right)}-6 \sum_{\substack{n=-\infty \\
(\bmod 7)}}^{\infty}(-1)^{n} q^{\frac{1}{2}\left(3 n^{2}+n\right)} \\
& =\sum_{n=-\infty}^{\infty}(-1)^{n} q^{\frac{1}{2}\left(3 n^{2}+n\right)}+7 q^{2} \sum_{n=-\infty}^{\infty}(-1)^{n} q^{\frac{1}{2}\left(147 n^{2}+49 n\right)} \\
& =(q ; q)_{\infty}+7 q^{2}\left(q^{49} ; q^{49}\right)_{\infty} .
\end{aligned}
$$

In the last step we have used Euler's identity (3.2). Substituting the above equation into (3.4) we obtain (1.19). This completes the proof of (1.19).

\section{The proofs of (1.20) and (1.21).}

We first prove (1.21) and then prove (1.20).

Let

$$
f(z)=\frac{\theta_{1}^{3}(z \mid q)}{\theta_{1}\left(z-\frac{\pi}{7} \mid q\right) \theta_{1}\left(z-\frac{2 \pi}{7} \mid q\right) \theta_{1}\left(z-\frac{4 \pi}{7} \mid q\right)} .
$$

Using (2.4) we can easily show that $f(z)$ is an elliptic functions with periods $\pi$ and $\pi \tau$. It has three simple poles $\frac{\pi}{7}, \frac{2 \pi}{7}$, and $\frac{4 \pi}{7}$ and no other poles.

Let $\operatorname{res}(f ; x)$ denote the residue of $f(z)$ at $x$. We have the following evaluations:

$$
\begin{aligned}
\operatorname{res}\left(f ; \frac{\pi}{7}\right) & =\lim _{z \rightarrow \frac{\pi}{7}}\left(z-\frac{\pi}{7}\right) f(z) \\
& =\lim _{z \rightarrow \frac{\pi}{7}} \frac{\left(z-\frac{\pi}{7}\right)}{\theta_{1}\left(z-\frac{\pi}{7} \mid q\right)} \times \lim _{z \rightarrow \frac{\pi}{7}} \frac{\theta_{1}^{3}(z \mid q)}{\theta_{1}\left(z-\frac{2 \pi}{7} \mid q\right) \theta_{1}\left(z-\frac{4 \pi}{7} \mid q\right)} .
\end{aligned}
$$

By L'Hôpital's rule,

$$
\lim _{z \rightarrow \frac{\pi}{7}} \frac{\left(z-\frac{\pi}{7}\right)}{\theta_{1}\left(z-\frac{\pi}{7} \mid q\right)}=\frac{1}{\theta_{1}^{\prime}(0 \mid q)}
$$

It is plain that

$$
\lim _{z \rightarrow \frac{\pi}{7}} \frac{\theta_{1}^{3}(z \mid q)}{\theta_{1}\left(z-\frac{2 \pi}{7} \mid q\right) \theta_{1}\left(z-\frac{4 \pi}{7} \mid q\right)}=\frac{\theta_{1}^{2}\left(\frac{\pi}{7} \mid q\right)}{\theta_{1}\left(\frac{3 \pi}{7} \mid q\right)} .
$$


Therefore we have

$$
\operatorname{res}\left(f ; \frac{\pi}{7}\right)=\frac{\theta_{1}^{2}\left(\frac{\pi}{7} \mid q\right)}{\theta_{1}^{\prime}(0 \mid q) \theta_{1}\left(\frac{3 \pi}{7} \mid q\right)} .
$$

In the same way we find that

$$
\begin{aligned}
& \operatorname{res}\left(f ; \frac{2 \pi}{7}\right)=-\frac{\theta_{1}^{2}\left(\frac{2 \pi}{7} \mid q\right)}{\theta_{1}^{\prime}(0 \mid q) \theta_{1}\left(\frac{\pi}{7} \mid q\right),} \\
& \operatorname{res}\left(f ; \frac{4 \pi}{7}\right)=\frac{\theta_{1}^{2}\left(\frac{3 \pi}{7} \mid q\right)}{\theta_{1}^{\prime}(0 \mid q) \theta_{1}\left(\frac{2 \pi}{7} \mid q\right)} .
\end{aligned}
$$

On the other hand, Lemma 2 gives

$$
\operatorname{res}\left(f ; \frac{\pi}{7}\right)+\operatorname{res}\left(f ; \frac{2 \pi}{7}\right)+\operatorname{res}\left(f ; \frac{4 \pi}{7}\right)=0 .
$$

Substituting (4.5)-(4.7) into the above equation we obtain (1.21).

We are now ready to prove (1.20). Letting

$$
a:=\frac{\theta_{1}\left(\frac{2 \pi}{7} \mid q\right)}{\theta_{1}\left(\frac{\pi}{7} \mid q\right)}, \quad b:=-\frac{\theta_{1}\left(\frac{3 \pi}{7} \mid q\right)}{\theta_{1}\left(\frac{2 \pi}{7} \mid q\right)}, \quad c:=\frac{\theta_{1}\left(\frac{\pi}{7} \mid q\right)}{\theta_{1}\left(\frac{3 \pi}{7} \mid q\right)},
$$

and recalling (1.3), we find that (1.19) can be rewritten as

$$
a+b+c=1+\rho(\tau) .
$$

Using (4.4) we find that (1.21) can be written as

$$
a b^{2}-a^{2}+c=0 \text {. }
$$

It is obvious that

$$
a b c=-1 .
$$

Multiplying (4.11) by $a^{-1}$ and $c$, respectively, and then using (4.12) in the resulting equations we find that

$$
\begin{aligned}
& b c^{2}-b^{2}+a=0, \\
& c a^{2}-c^{2}+b=0 .
\end{aligned}
$$

Denote

$$
Q:=a b+b c+c a, \quad P:=a+b+c=1+\rho(\tau), \quad R:=a b c=-1 .
$$

Multiplying (4.11) by $a,(4.13)$ by $b$, and (4.14) by $c$ and then adding the resulting equations we find that

$$
\left(a^{2} b^{2}+b^{2} c^{2}+c^{2} a^{2}\right)-\left(a^{3}+b^{3}+c^{3}\right)+a b+b c+c a=0 .
$$

Using the theory of elementary symmetric polynomials, we readily find that the above equation can be rewritten as

$$
Q^{2}+(3 \rho(\tau)+4) Q-\left(\rho^{3}(\tau)+3 \rho^{2}(\tau)+\rho(\tau)-4\right)=0 .
$$


Solving the above equation for $Q$, we obtain

$$
Q=-\frac{1}{2}(3 \rho(\tau)+4)-\frac{1}{2} \sqrt{4 \rho^{3}(\tau)+21 \rho^{2}(\tau)+28 \rho(\tau)} .
$$

Noting the definitions of $a, b$, and $c$, (4.9), we find that (4.18) is (1.20).

\section{The proofs of (1.22) and (1.23).}

Using (2.6) and (4.9) we readily find that

$$
\begin{aligned}
& y_{1}:=a^{3} b=-\sqrt{7} \eta^{2}(\tau) \eta(7 \tau) \frac{\theta_{1}\left(\frac{2 \pi}{7} \mid q\right)}{\theta_{1}^{4}\left(\frac{\pi}{7} \mid q\right)}, \\
& y_{2}:=b^{3} c=-\sqrt{7} \eta^{2}(\tau) \eta(7 \tau) \frac{\theta_{1}\left(\frac{3 \pi}{7} \mid q\right)}{\theta_{1}^{4}\left(\frac{2 \pi}{7} \mid q\right)}, \\
& y_{3}:=c^{3} a=\sqrt{7} \eta^{2}(\tau) \eta(7 \tau) \frac{\theta_{1}\left(\frac{\pi}{7} \mid q\right)}{\theta_{1}^{4}\left(\frac{3 \pi}{7} \mid q\right)} .
\end{aligned}
$$

From (4.11)-(4.14) and some straightforward evaluations we find that

$$
\begin{aligned}
y_{1} y_{2} & =-y_{1}-1, \\
y_{2} y_{3} & =-y_{2}-1, \\
y_{3} y_{1} & =-y_{3}-1, \\
y_{1} y_{2} y_{3} & =1 .
\end{aligned}
$$

We now compute $y_{1}+y_{2}+y_{3}$ and $y_{1} y_{2}+y_{2} y_{3}+y_{3} y_{1}$. Noting (4.12) and (4.15), we have the evaluation

$$
\begin{aligned}
P Q & =(a+b+c)(a b+b c+c a) \\
& =a c^{2}+c b^{2}+b a^{2}+a b^{2}+b c^{2}+c a^{2}-3 .
\end{aligned}
$$

Adding (4.11), (4.13), and (4.14), we find that

$$
\begin{aligned}
a b^{2}+b c^{2}+c a^{2} & =a^{2}+b^{2}+c^{2}-a-b-c \\
& =(a+b+c)^{2}-2(a b+b c+c a)-a-b-c \\
& =P^{2}-2 Q-P .
\end{aligned}
$$

Substituting the above equation into (5.8), we find that

$$
a c^{2}+c b^{2}+b a^{2}=-P^{2}+P Q+P+2 Q+3 .
$$

Using (4.11), (4.13), (4.14), and the above equation, we readily find that

$$
\begin{aligned}
a b^{3}+b c^{3}+c a^{3} & =a\left(c^{2}-b\right)+b\left(a^{2}-c\right)+c\left(b^{2}-a\right) \\
& =a c^{2}+c b^{2}+b a^{2}-a b-b c-c a \\
& =-P^{2}+P Q+P+Q+3 .
\end{aligned}
$$


Employing (4.11), (4.12), (4.13), (4.14), and the above equation, we find that

$$
\begin{aligned}
a^{3} b+b^{3} c+c^{3} a= & \left(a^{2}+b^{2}+c^{2}\right)(a b+b c+c a) \\
& -a b^{3}-b c^{3}-c a^{3}+a+b+c \\
= & \left(P^{2}-2 Q\right) Q+P^{2}-P Q-P-Q-3+P \\
= & P^{2} Q+P^{2}-2 Q^{2}-P Q-Q-3 .
\end{aligned}
$$

Therefore, by using Lemma 4, (4.10), (4.18), and the definitions of $y_{1}, y_{2}$, and $y_{3}$, we obtain

$$
\begin{aligned}
& y_{1}+y_{2}+y_{3}=a^{3} b+b^{3} c+c^{3} a \\
&=P^{2} Q+P^{2}-P Q-2 Q^{2}-Q-3 \\
&=\left(\rho^{2}(\tau)+7 \rho(\tau)+7\right) Q-2 \rho^{3}(\tau)-5 \rho^{2}(\tau)+6 \\
&=-\frac{1}{2}\left(\rho^{2}(\tau)+7 \rho(\tau)+7\right)\left(3 \rho(\tau)+4+\sqrt{4 \rho^{3}(\tau)+21 \rho^{2}(\tau)+28 \rho(\tau)}\right) \\
&-2 \rho^{3}(\tau)-5 \rho^{2}(\tau)+6 \\
&=-\frac{1}{2}\left(\rho^{2}(\tau)+7 \rho(\tau)+7\right) \sqrt{4 \rho^{3}(\tau)+21 \rho^{2}(\tau)+28 \rho(\tau)} \\
&-\frac{1}{2}\left(7 \rho^{3}(\tau)+35 \rho^{2}(\tau)+49 \rho(\tau)\right)-8 \\
&=-8-49 \frac{\eta^{4}(7 \tau)}{\eta^{4}(\tau)}=-8-49 h(\tau) .
\end{aligned}
$$

The above equation is equivalent to (1.22).

Adding (5.4), (5.5), and (5.6) and then using the above equation we immediately have

$$
\begin{aligned}
y_{1} y_{2}+y_{2} y_{3}+y_{3} y_{1} & =-\left(y_{1}+y_{2}+y_{3}\right)-3 \\
& =5+49 \frac{\eta^{4}(7 \tau)}{\eta^{4}(\tau)}=5+49 h(\tau) .
\end{aligned}
$$

The above equation is equivalent to (1.23).

\section{The proofs of (1.24) and (1.25).}

Multiplying (4.11) by $a b,(4.13)$ by $b c,(4.14)$ by $a c$, and noting the definitions of $y_{1}, y_{2}$, and $y_{3}$, we find that

$$
a^{2} b^{3}=y_{1}+1, \quad b^{2} c^{3}=y_{2}+1, \quad c^{2} a^{3}=y_{3}+1 .
$$


Multiplying (4.11) by $b^{3},(4.13)$ by $c^{3},(4.14)$ by $a^{3}$, and using the definitions of $y_{1}, y_{2}$, and $y_{3}$, we obtain

$$
a b^{5}=a^{2} b^{3}-y_{2}, \quad b c^{5}=b^{2} c^{3}-y_{3}, \quad c a^{5}=c^{2} a^{3}-y_{1} .
$$

Combining (6.1) and (6.2) we have

$$
a b^{5}=y_{1}-y_{2}+1, \quad b c^{5}=y_{2}-y_{3}+1, \quad c a^{5}=y_{3}-y_{1}+1 .
$$

Multiplying (4.11) by $a^{5},(4.13)$ by $b^{5}$ and (4.14) by $c^{5}$, we find that

$$
a^{7}=a^{5} c+y_{1}^{2}, \quad b^{7}=b^{5} a+y_{2}^{2}, \quad c^{7}=c^{5} b+y_{3}^{2} .
$$

From (6.3) and (6.4) we find the following relations:

$$
a^{7}=y_{1}^{2}-y_{1}+y_{3}+1, \quad b^{7}=y_{2}^{2}-y_{2}+y_{1}+1, \quad c^{7}=y_{3}^{2}-y_{3}+y_{2}+1 .
$$

Using the above relations, (5.13), and (5.14), we immediately have

$$
\begin{aligned}
& a^{7}+b^{7}+c^{7} \\
& =y_{1}^{2}+y_{2}^{2}+y_{3}^{2}+3 \\
& =\left(y_{1}+y_{2}+y_{3}\right)^{2}-2\left(y_{1} y_{2}+y_{2} y_{3}+y_{3} y_{1}\right)+3 \\
& =(8+49 h(\tau))^{2}-2(5+49 h(\tau))+3 \\
& =57+2 \times 7^{3} h(\tau)+7^{4} h^{2}(\tau) .
\end{aligned}
$$

The above equation is equivalent to (1.24).

By using (6.5) and (5.4)-(5.7) we find that

$$
a^{7} b^{7}=y_{1}\left(y_{1}+1\right)^{2}, \quad b^{7} c^{7}=y_{2}\left(y_{2}+1\right)^{2}, \quad c^{7} a^{7}=y_{3}\left(y_{3}+1\right)^{2} .
$$

Adding the three equations together in (6.7) and then using (5.4)-(5.7), (5.13), and (5.14), we obtain

$$
\begin{aligned}
a^{7} & b^{7}+b^{7} c^{7}+c^{7} a^{7} \\
= & y_{1}\left(y_{1}+1\right)^{2}+y_{2}\left(y_{2}+1\right)^{2}+y_{3}\left(y_{3}+1\right)^{2} \\
= & \left(y_{1}+y_{2}+y_{3}\right)^{3}-3\left(y_{1}+y_{2}+y_{3}\right)\left(y_{1} y_{2}+y_{2} y_{3}+y_{3} y_{1}\right) \\
& \quad+3 y_{1} y_{2} y_{3}+2\left(y_{1}+y_{2}+y_{3}\right)^{2}-4\left(y_{1} y_{2}+y_{2} y_{3}+y_{3} y_{1}\right) \\
& \quad+y_{1}+y_{2}+y_{3} \\
= & \left(y_{1}+y_{2}+y_{3}\right)^{3}+5\left(y_{1}+y_{2}+y_{3}\right)^{2}+14\left(y_{1}+y_{2}+y_{3}\right)+15 \\
= & -289-18 \times 7^{3} h(\tau)-19 \times 7^{4} h^{2}(\tau)-7^{6} h^{3}(\tau) .
\end{aligned}
$$

The above equation is equivalent to (1.25). 


\section{The proofs of (1.26), (1.27) and (1.28).}

Multiplying (4.11) by $a^{4} b^{2}$, (4.13) by $b^{4} c^{2}$, (4.14) by $a^{2} c^{4}$, and using (5.1)(5.4), we find that

$$
a^{5} b^{4}=y_{1}^{2}+y_{1}, \quad b^{5} c^{4}=y_{2}^{2}+y_{2}, \quad c^{5} a^{4}=y_{3}^{2}+y_{3} .
$$

Therefore we have

$$
\begin{aligned}
a^{5} b^{4}+b^{5} c^{4}+c^{5} a^{4}= & y_{1}^{2}+y_{1}+y_{2}^{2}+y_{2}+y_{3}^{2}+y_{3} \\
= & \left(y_{1}+y_{2}+y_{3}\right)^{2}-2\left(y_{1} y_{2}+y_{2} y_{3}+y_{3} y_{1}\right) \\
& +y_{1}+y_{2}+y_{3} .
\end{aligned}
$$

Substituting (5.13) and (5.14) into the above equation we obtain

$$
a^{5} b^{4}+b^{5} c^{4}+c^{5} a^{4}=46+13 \times 49 h(\tau)+49^{2} h^{2}(\tau) .
$$

The above equation is the same as (1.26).

Now we prove (1.27). By a direct evaluation,

$$
\begin{aligned}
& \left(x_{1}+x_{2}+x_{3}\right)^{3} \\
& =x_{1}^{3}+x_{2}^{3}+x_{3}^{3}+6 x_{1} x_{2} x_{3} \\
& \quad+3 x_{1}^{2} x_{2}+3 x_{1}^{2} x_{3}+3 x_{2}^{2} x_{1}+3 x_{2}^{2} x_{3}+3 x_{3}^{2} x_{1}+3 x_{3}^{3} x_{2} .
\end{aligned}
$$

Taking $x_{1}=\sqrt[3]{y_{1}^{2} y_{2}}, x_{2}=\sqrt[3]{y_{2}^{2} y_{3}}$, and $x_{3}=\sqrt[3]{y_{3}^{2} y_{1}}$ and using (5.4)-(5.7), we obtain

$$
\begin{aligned}
& \left(\sqrt[3]{y_{1}^{2} y_{2}}+\sqrt[3]{y_{2}^{2} y_{3}}+\sqrt[3]{y_{3}^{2} y_{1}}\right)^{3} \\
= & y_{1}^{2} y_{2}+y_{2}^{2} y_{3}+y_{3}^{2} y_{1}+3\left(y_{1}+y_{2}+y_{3}\right) \\
& +3\left(y_{1} y_{2}+y_{2} y_{3}+y_{3} y_{4}\right)+6 \\
= & -y_{1}\left(y_{1}+1\right)-y_{2}\left(y_{2}+1\right)-y_{3}\left(y_{3}+1\right) \\
& +3\left(y_{1}+y_{2}+y_{3}\right)+3\left(y_{1} y_{2}+y_{2} y_{3}+y_{3} y_{4}\right)+6 \\
= & -y_{1}^{2}-y_{2}^{2}-y_{3}^{2}+2\left(y_{1}+y_{2}+y_{3}\right) \\
& +3\left(y_{1} y_{2}+y_{2} y_{3}+y_{3} y_{4}\right)+6 \\
= & -\left(y_{1}+y_{2}+y_{3}\right)^{2}-3\left(y_{1}+y_{2}+y_{3}\right)-9 \\
= & -49\left(1+13 h(\tau)+49 h^{2}(\tau)\right) .
\end{aligned}
$$

Noting the definitions of $y_{1}, y_{2}$, and $y_{3}$, we find that the above equation is equivalent to (1.27).

Finally we prove (1.28). Denote

$$
\Delta:=-8-49 h(\tau)
$$


Then (5.13) and (5.14) can be written in the following forms, respectively:

$$
\begin{aligned}
y_{1}+y_{2}+y_{3} & =\Delta \\
y_{1} y_{2}+y_{2} y_{3}+y_{4} y_{5} & =-\Delta-3 .
\end{aligned}
$$

By (5.4)-(5.7), (7.7), and (7.8),

$$
\begin{aligned}
& y_{1}^{2}+y_{2}^{2}+y_{3}^{2}=\Delta^{2}+2 \Delta+6, \\
& y_{1}^{3}+y_{2}^{3}+y_{3}^{3}=\Delta^{3}+3 \Delta^{2}+9 \Delta+3, \\
& y_{1}^{4}+y_{2}^{4}+y_{3}^{4}=\Delta^{4}+4 \Delta^{3}+14 \Delta^{2}+16 \Delta+18, \\
& y_{1}^{5}+y_{2}^{5}+y_{3}^{5}=\Delta^{5}+5 \Delta^{4}+20 \Delta^{3}+35 \Delta^{2}+50 \Delta+15 .
\end{aligned}
$$

Taking $x_{1}=\sqrt[3]{y_{1}^{5} y_{2}}, x_{2}=\sqrt[3]{y_{2}^{5} y_{3}}$, and $x_{3}=\sqrt[3]{y_{3}^{5} y_{1}}$ in (7.4) and using (5.4)-(5.7), we obtain

$$
\begin{aligned}
& \left(\sqrt[3]{y_{1}^{5} y_{2}}+\sqrt[3]{y_{2}^{5} y_{3}}+\sqrt[3]{y_{3}^{5} y_{1}}\right)^{3} \\
= & y_{1}^{5} y_{2}+y_{2}^{5} y_{3}+y_{3}^{5} y_{1}+3\left(y_{1}^{3} y_{3}+y_{3}^{3} y_{2}+y_{2}^{3} y_{1}\right) \\
& +3\left(y_{1}^{3} y_{2}^{2}+y_{2}^{3} y_{3}^{2}+y_{3}^{3} y_{1}^{2}\right)+6 \\
= & -\left(y_{1}^{5}+y_{2}^{5}+y_{3}^{5}\right)-\left(y_{1}^{4}+y_{2}^{4}+y_{3}^{4}\right) \\
& +3\left(y_{1}^{3}+y_{2}^{3}+y_{3}^{3}\right)+3\left(y_{1}^{2}+y_{2}^{2}+y_{3}^{2}\right)+3\left(y_{1}+y_{2}+y_{3}\right)-3 \\
= & -\left(\Delta^{2}+3 \Delta+9\right)(\Delta+1)^{3} \\
= & 7^{5}\left(1+13 h(\tau)+49 h^{2}(\tau)\right)(1+7 h(\tau)) .
\end{aligned}
$$

Substituting (5.1)-(5.3) and (7.6) into the above equation, we obtain (1.28).

\section{The proofs of (1.15), (1.16) and (1.17).}

We recall the following identity (see, for example, $[\mathbf{2 0}]$ ):

$$
\begin{aligned}
& \cot ^{2} y-\cot ^{2} x+8 \sum_{n=1}^{\infty} \frac{n q^{n}}{1-q^{n}}(\cos 2 n x-\cos 2 n y) \\
& =\theta_{1}^{\prime}(0 \mid q)^{2} \frac{\theta_{1}(x-y \mid q) \theta_{1}(x+y \mid q)}{\theta_{1}^{2}(x \mid q) \theta_{1}^{2}(x \mid q)} .
\end{aligned}
$$

Dividing both sides of this equation by $x-y$ and then letting $y \rightarrow x$, we get

$$
2 \cot x\left(1+\cot ^{2} x\right)-16 \sum_{n=1}^{\infty} \frac{n^{2} q^{n}}{1-q^{n}} \sin 2 n x=\theta_{1}^{\prime}(0 \mid q)^{3} \frac{\theta_{1}(2 x \mid q)}{\theta_{1}^{4}(x \mid q)} .
$$


Taking $x=\frac{\pi}{7}, \frac{2 \pi}{7}$, and $-\frac{3 \pi}{7}$, respectively, in the above equation and then adding the resulting equations we get

$$
s-16 \sum_{n=1}^{\infty} s(n) \frac{n^{2} q^{n}}{1-q^{n}}=\theta_{1}^{\prime}(0 \mid q)^{3}\left(\frac{\theta_{1}\left(\frac{2 \pi}{7} \mid q\right)}{\theta_{1}^{4}\left(\frac{\pi}{7} \mid q\right)}-\frac{\theta_{1}\left(\frac{\pi}{7} \mid q\right)}{\theta_{1}^{4}\left(\frac{3 \pi}{7} \mid q\right)}+\frac{\theta_{1}\left(\frac{3 \pi}{7} \mid q\right)}{\theta_{1}^{4}\left(\frac{2 \pi}{7} \mid q\right)}\right) .
$$

Here

$$
\begin{aligned}
s= & 2 \cot \frac{\pi}{7}\left(1+\cot ^{3} \frac{\pi}{7}\right)+2 \cot \frac{2 \pi}{7}\left(1+\cot ^{3} \frac{2 \pi}{7}\right) \\
& -2 \cot \frac{3 \pi}{7}\left(1+\cot ^{3} \frac{3 \pi}{7}\right),
\end{aligned}
$$

$$
s(n)=\sin \frac{2 n \pi}{7}+\sin \frac{4 n \pi}{7}-\sin \frac{6 n \pi}{7} .
$$

Setting $q=0$ in (8.3) and then using (1.32) we have

$$
s=\frac{\sin (2 \pi / 7)}{\sin ^{4}(\pi / 7)}-\frac{\sin (\pi / 7)}{\sin ^{4}(3 \pi / 7)}+\frac{\sin (3 \pi / 7)}{\sin ^{4}(2 \pi / 7)}=\frac{64}{7} \sqrt{7} .
$$

From [13, p. 145, Equation (7.18)] we know that

$$
s(n)=\sin \frac{2 n \pi}{7}+\sin \frac{4 n \pi}{7}-\sin \frac{6 n \pi}{7}=\frac{\sqrt{7}}{2}\left(\frac{n}{7}\right) .
$$

Substituting (8.6) and (8.7) into (8.3) and then using (1.22) in the resuting equation we obtain (1.15).

To prove (1.16), we recall the identity of Mccullogh and L.-C. Shen (see Lemma 3)

$$
\begin{aligned}
& \frac{\theta_{1}^{\prime}}{\theta_{1}}(x \mid q)+\frac{\theta_{1}^{\prime}}{\theta_{1}}(y \mid q)+\frac{\theta_{1}^{\prime}}{\theta_{1}}(z \mid q)-\frac{\theta_{1}^{\prime}}{\theta_{1}}(x+y+z \mid q) \\
& =\theta_{1}^{\prime}(0 \mid q) \frac{\theta_{1}(x+y \mid q) \theta_{1}(y+z \mid q) \theta_{1}(z+x \mid q)}{\theta_{1}(x \mid q) \theta_{1}(y \mid q) \theta_{1}(z \mid q) \theta_{1}(x+y+z \mid q)} .
\end{aligned}
$$

Taking $(x, y, z)=\left(\frac{\pi}{7},-\frac{3 \pi}{7},-\frac{3 \pi}{7}\right),\left(\frac{\pi}{7},-\frac{2 \pi}{7},-\frac{2 \pi}{7}\right)$, and $\left(\frac{\pi}{7}, \frac{\pi}{7}, \frac{2 \pi}{7}\right)$, respectively, in the above equation we obtain

$$
\begin{aligned}
& \frac{\theta_{1}^{\prime}}{\theta_{1}}\left(\frac{\pi}{7} \mid q\right)-\frac{\theta_{1}^{\prime}}{\theta_{1}}\left(\frac{2 \pi}{7} \mid q\right)-2 \frac{\theta_{1}^{\prime}}{\theta_{1}}\left(\frac{3 \pi}{7} \mid q\right)=\theta_{1}^{\prime}(0 \mid q) \frac{\theta_{1}\left(\frac{2 \pi}{7} \mid q\right)}{\theta_{1}^{2}\left(\frac{3 \pi}{7} \mid q\right)} \\
& \frac{\theta_{1}^{\prime}}{\theta_{1}}\left(\frac{\pi}{7} \mid q\right)-2 \frac{\theta_{1}^{\prime}}{\theta_{1}}\left(\frac{2 \pi}{7} \mid q\right)+\frac{\theta_{1}^{\prime}}{\theta_{1}}\left(\frac{3 \pi}{7} \mid q\right)=\theta_{1}^{\prime}(0 \mid q) \frac{\theta_{1}\left(\frac{\pi}{7} \mid q\right)}{\theta_{1}^{2}\left(\frac{2 \pi}{7} \mid q\right)} \\
& 2 \frac{\theta_{1}^{\prime}}{\theta_{1}}\left(\frac{\pi}{7} \mid q\right)+\frac{\theta_{1}^{\prime}}{\theta_{1}}\left(\frac{2 \pi}{7} \mid q\right)+\frac{\theta_{1}^{\prime}}{\theta_{1}}\left(\frac{3 \pi}{7} \mid q\right)=\theta_{1}^{\prime}(0 \mid q) \frac{\theta_{1}\left(\frac{3 \pi}{7} \mid q\right)}{\theta_{1}^{2}\left(\frac{\pi}{7} \mid q\right)} .
\end{aligned}
$$


Adding (8.9), (8.10), and (8.11) gives

$$
\begin{aligned}
& 2\left(\cot \frac{\pi}{7}+\cot \frac{2 \pi}{7}-\cot \frac{3 \pi}{7}\right) \\
& \quad+8 \sum_{n=1}^{\infty} \frac{q^{n}}{1-q^{n}}\left(\sin \frac{2 n \pi}{7}+\sin \frac{4 n \pi}{7}-\sin \frac{6 n \pi}{7}\right) \\
& =\theta_{1}^{\prime}(0 \mid q)\left(\frac{\theta_{1}\left(\frac{3 \pi}{7} \mid q\right)}{\theta_{1}^{2}\left(\frac{\pi}{7} \mid q\right)}-\frac{\theta_{1}\left(\frac{\pi}{7} \mid q\right)}{\theta_{1}^{2}\left(\frac{2 \pi}{7} \mid q\right)}+\frac{\theta_{1}\left(\frac{2 \pi}{7} \mid q\right)}{\theta_{1}^{2}\left(\frac{3 \pi}{7} \mid q\right)}\right) .
\end{aligned}
$$

Setting $q=0$ and then using (1.35), we obtain

$$
\cot \frac{\pi}{7}+\cot \frac{2 \pi}{7}-\cot \frac{3 \pi}{7}=\sqrt{7}
$$

Substituting (8.7), (8.13), and (1.27) into the above equation we obtain (1.16).

To prove (1.14), we construct the following elliptic function:

$$
f(z):=\frac{\theta_{1}\left(z+\frac{\pi}{7} \mid q\right) \theta_{1}\left(z+\frac{2 \pi}{7} \mid q\right) \theta_{1}\left(z-\frac{3 \pi}{7} \mid q\right)}{\theta_{1}^{3}(z \mid q)} .
$$

By using (2.4), it is easy to check that $f(z)$ is an elliptic function with periods $\pi$ and $\pi \tau$. Also, $f(z)$ has only one pole at 0 , and its order is 3 . We now compute res $(f ; 0)$.

It is plain that

$$
\operatorname{res}(f ; 0)=\frac{1}{2}\left[\frac{d^{2}\left(z^{3} f(z)\right)}{d^{2} z}\right]_{z=0} .
$$

Set

$$
F(z):=z^{3} f(z), \quad \phi(z)=\frac{F^{\prime}(z)}{F(z)} .
$$

By logarithmic differentitation we easily find that

$$
\operatorname{res}(f ; 0)=\frac{1}{2}\left[\frac{d^{2}\left(z^{3} f(z)\right)}{d^{2} z}\right]_{z=0}=\frac{1}{2} F(0)\left(\phi(0)^{2}+\phi^{\prime}(0)\right) .
$$

Using (2.10) we find that

$$
\begin{aligned}
\phi(z)= & \frac{z}{3}-3 \frac{\theta_{1}^{\prime}}{\theta_{1}}(z \mid q)+\frac{\theta_{1}^{\prime}}{\theta_{1}}\left(z+\frac{\pi}{7} \mid q\right) \\
& +\frac{\theta_{1}^{\prime}}{\theta_{1}}\left(z+\frac{2 \pi}{7} \mid q\right)+\frac{\theta_{1}^{\prime}}{\theta_{1}}\left(z-\frac{3 \pi}{7} \mid q\right) \\
= & L(\tau) z+\frac{\theta_{1}^{\prime}}{\theta_{1}}\left(z+\frac{\pi}{7} \mid q\right) \\
& +\frac{\theta_{1}^{\prime}}{\theta_{1}}\left(z+\frac{2 \pi}{7} \mid q\right)+\frac{\theta_{1}^{\prime}}{\theta_{1}}\left(z-\frac{3 \pi}{7} \mid q\right)+O\left(z^{3}\right) .
\end{aligned}
$$


Setting $z=0$ and then using (8.7) and (8.13), we obtain

$$
\begin{aligned}
\phi(0)= & \frac{\theta_{1}^{\prime}}{\theta_{1}}\left(\frac{\pi}{7} \mid q\right)+\frac{\theta_{1}^{\prime}}{\theta_{1}}\left(\frac{2 \pi}{7} \mid q\right)-\frac{\theta_{1}^{\prime}}{\theta_{1}}\left(\frac{3 \pi}{7} \mid q\right) \\
= & \left(\cot \frac{\pi}{7}+\cot \frac{2 \pi}{7}-\cot \frac{3 \pi}{7}\right) \\
& +4 \sum_{n=1}^{\infty} \frac{q^{n}}{1-q^{n}}\left(\sin \frac{2 n \pi}{7}+\sin \frac{4 n \pi}{7}-\sin \frac{6 n \pi}{7}\right) \\
= & \sqrt{7}\left(1+2 \sum_{n=1}^{\infty}\left(\frac{n}{7}\right) \frac{q^{n}}{1-q^{n}}\right) .
\end{aligned}
$$

Differentiating (8.18) with respect to $z$, setting $z=0$, and using (2.14), we find that

$$
\begin{aligned}
\phi^{\prime}(0) & =L(\tau)+\left(\frac{\theta_{1}^{\prime}}{\theta_{1}}\right)^{\prime}\left(\frac{\pi}{7} \mid q\right)+\left(\frac{\theta_{1}^{\prime}}{\theta_{1}}\right)^{\prime}\left(\frac{2 \pi}{7} \mid q\right)+\left(\frac{\theta_{1}^{\prime}}{\theta_{1}}\right)^{\prime}\left(\frac{3 \pi}{7} \mid q\right) \\
& =-7\left(1+4 \sum_{n=1}^{\infty} \frac{n q^{n}}{1-q^{n}}-28 \sum_{n=1}^{\infty} \frac{n q^{7 n}}{1-q^{7 n}}\right) .
\end{aligned}
$$

Note that

$$
F(0)=-\frac{\theta_{1}\left(\frac{\pi}{7} \mid q\right) \theta_{1}\left(\frac{2 \pi}{7} \mid q\right) \theta_{1}\left(\frac{3 \pi}{7} \mid q\right)}{\theta_{1}^{\prime}(0 \mid q)^{3}} \neq 0 .
$$

Substituting (8.19) and (8.20) into (8.17) and using Lemma 2, we find that

$$
1+4 \sum_{n=1}^{\infty} \frac{n q^{n}}{1-q^{n}}-28 \sum_{n=1}^{\infty} \frac{n q^{7 n}}{1-q^{7 n}}=\left(1+2 \sum_{n=1}^{\infty}\left(\frac{n}{7}\right) \frac{q^{n}}{1-q^{n}}\right)^{2} .
$$

Combining (1.16) and (8.22) we obtain (1.17).

\section{The proofs of (1.8) and (1.9).}

To prove (1.8) and (1.9), we introduce the function

$$
f(z)=\frac{\theta_{1}(2 z \mid q) \theta_{1}(3 z \mid q)}{\theta_{1}^{6}(z \mid q) \theta_{1}\left(7 z \mid q^{7}\right)}
$$

By using (2.4) we readily verify that $f(z)$ is an elliptic function with periods $\pi$ and $\pi \tau$. The poles of $f(z)$ are 0 and $\frac{\pi}{7}, \frac{2 \pi}{7}, \ldots, \frac{6 \pi}{7}$. Furthermore, $\frac{\pi}{7}, \frac{2 \pi}{7}, \ldots, \frac{6 \pi}{7}$ are simple poles and 0 is a pole of order 5 .

From Lemma 2, we have

$$
\operatorname{res}(f ; 0)+\sum_{k=1}^{6} \operatorname{res}\left(f ; \frac{k \pi}{7}\right)=0 .
$$


Now,

$(9.3)$

$$
\begin{aligned}
\operatorname{res}\left(f ; \frac{\pi}{7}\right) & =\lim _{z \rightarrow \frac{\pi}{7}}\left(z-\frac{\pi}{7}\right) f(z) \\
& =-\frac{\theta_{1}\left(\frac{2 \pi}{7} \mid q\right) \theta_{1}\left(\frac{3 \pi}{7} \mid q\right)}{7 \theta_{1}^{\prime}\left(0 \mid q^{7}\right) \theta_{1}^{6}\left(\frac{\pi}{7} \mid q\right)}=-\frac{1}{2 \sqrt{7}} \frac{\eta^{2}(\tau)}{\eta^{2}(7 \tau)} \theta_{1}^{-7}\left(\frac{\pi}{7} \mid q\right)
\end{aligned}
$$

and we also find that

$$
\operatorname{res}\left(f ; \frac{6 \pi}{7}\right)=\operatorname{res}\left(f ; \frac{\pi}{7}\right)=-\frac{1}{2 \sqrt{7}} \frac{\eta^{2}(\tau)}{\eta^{2}(7 \tau)} \theta_{1}^{-7}\left(\frac{\pi}{7} \mid q\right) .
$$

In the same way we find that

$$
\begin{aligned}
& \operatorname{res}\left(f ; \frac{2 \pi}{7}\right)=\operatorname{res}\left(f ; \frac{5 \pi}{7}\right)=\frac{1}{2 \sqrt{7}} \frac{\eta^{2}(\tau)}{\eta^{2}(7 \tau)} \theta_{1}^{-7}\left(\frac{2 \pi}{7} \mid q\right), \\
& \operatorname{res}\left(f ; \frac{3 \pi}{7}\right)=\operatorname{res}\left(f ; \frac{4 \pi}{7}\right)=\frac{1}{2 \sqrt{7}} \frac{\eta^{2}(\tau)}{\eta^{2}(7 \tau)} \theta_{1}^{-7}\left(\frac{3 \pi}{7} \mid q\right) .
\end{aligned}
$$

To compute res $(f ; 0)$, we define

$$
F(z):=z^{5} f(z), \quad \phi(z):=\frac{F^{\prime}(z)}{F(z)} .
$$

It is plain that

$$
F(0)=\frac{6}{7 \theta_{1}^{\prime}\left(0 \mid q^{7}\right) \theta_{1}^{\prime}(0 \mid q)^{4}}=\frac{3}{112 \eta^{3}(7 \tau) \eta^{12}(\tau)} .
$$

By an elementary calculation,

$$
\begin{aligned}
& \operatorname{res}(f ; 0)=\frac{1}{24}\left[F^{(4)}(z)\right]_{z=0} \\
& =\frac{F(0)}{24}\left(\phi^{4}(0)+6 \phi^{2}(0) \phi^{\prime}(0)+4 \phi(0) \phi^{\prime \prime}(0)+3 \phi^{\prime}(0)^{2}+\phi^{\prime \prime \prime}(0)\right) .
\end{aligned}
$$

Using (2.10), we find that

$$
\begin{aligned}
\phi(z)= & \frac{5}{z}-6 \frac{\theta_{1}^{\prime}}{\theta_{1}}(z \mid q)+2 \frac{\theta_{1}^{\prime}}{\theta_{1}}(2 z \mid q)+3 \frac{\theta_{1}^{\prime}}{\theta_{1}}(3 z \mid q)-7 \frac{\theta_{1}^{\prime}}{\theta_{1}}\left(7 z \mid q^{7}\right) \\
= & \frac{7}{3}(7 L(7 \tau)-L(\tau)) z \\
& +\frac{7}{45}(343 M(7 \tau)-13 M(\tau)) z^{3}+O\left(z^{5}\right) .
\end{aligned}
$$

This yields

$$
\begin{aligned}
\phi^{\prime}(0) & =\frac{7}{3}(7 L(7 \tau)-L(\tau))=14 A(\tau), \quad \phi(0)=0, \quad \phi^{\prime \prime}(0)=0 \\
\phi^{\prime \prime \prime}(0) & =\frac{14}{15}(343 M(7 \tau)-13 M(\tau)) .
\end{aligned}
$$


Substituting the above equations into (9.9) we arrive at

$$
\begin{aligned}
\operatorname{res}(f ; 0)= & \frac{1}{960} \eta^{-3}(7 \tau) \eta^{-12}(\tau) \\
& \cdot\left(630 A^{2}(\tau)+343 M(7 \tau)-13 M(\tau)\right) .
\end{aligned}
$$

Substituting (9.3)-(9.6) and (9.13) into (9.2) we obtain

$$
\begin{aligned}
& 630 A^{2}(\tau)+343 M(7 \tau)-13 M(\tau) \\
& =\frac{960}{\sqrt{7}} \eta^{14}(\tau) \eta(7 \tau)\left(\theta_{1}^{-7}\left(\frac{\pi}{7} \mid q\right)-\theta_{1}^{-7}\left(\frac{2 \pi}{7} \mid q\right)-\theta_{1}^{-7}\left(\frac{3 \pi}{7} \mid q\right)\right) .
\end{aligned}
$$

Substituting (1.28) into (9.13) we obtain the following interesting result:

Lemma 8. We have

$$
\begin{aligned}
& 630 A^{2}(\tau)+343 M(7 \tau)-13 M(\tau) \\
& =960 k(\tau)^{4 / 3}(1+7 h(\tau))\left(1+13 h(\tau)+49 h^{2}(\tau)\right)^{1 / 3} .
\end{aligned}
$$

From [1, pp. 24, 48, 69] we know that

$$
\begin{aligned}
\eta(-1 / \tau) & =\sqrt{-i \tau} \eta(\tau), \\
L(-1 / \tau) & =-\frac{6 \tau i}{\pi}+\tau^{2} L(\tau), \\
M(-1 / \tau) & =\tau^{4} M(\tau), \\
N(-1 / \tau) & =\tau^{6} N(\tau) .
\end{aligned}
$$

It follows that

$$
\begin{aligned}
\eta(-1 / 7 \tau) & =\sqrt{-7 i \tau} \eta(7 \tau), \\
A(-1 / 7 \tau) & =-7 \tau^{2} A(\tau), \\
M(-1 / 7 \tau) & =(7 \tau)^{4} M(7 \tau), \\
N(-1 / 7 \tau) & =(7 \tau)^{6} N(7 \tau), \\
h(-1 / 7 \tau) & =7^{-2} h^{-1}(\tau) .
\end{aligned}
$$

Replacing $\tau$ by $-1 / 7 \tau$ in (9.14) and then using (9.20), (9.21), and (9.23) in the resulting equation we deduce that:

Lemma 9. We have

$$
\begin{aligned}
& 90 A^{2}(\tau)-91 M(7 \tau)+M(\tau) \\
& =960 k(\tau)^{4 / 3}\left(7 h(\tau)+h^{2}(\tau)\right)\left(1+13 h(\tau)+49 h^{2}(\tau)\right)^{1 / 3} .
\end{aligned}
$$

By solving the linear system of equations, (9.14) and (9.24), for $M(\tau)$ and $M(7 \tau)$ we deduce the following theorem: 
Theorem 10. We have

$$
\begin{gathered}
7 M(7 \tau)=15 A^{2}(\tau)-8 k(\tau)^{4 / 3}\left(1+20 h(\tau)+91 h^{2}(\tau)\right) \\
\cdot\left(1+13 h(\tau)+49 h^{2}(\tau)\right)^{1 / 3} \\
M(\tau)=105 A^{2}(\tau)-8 k(\tau)^{4 / 3}\left(13+140 h(\tau)+343 h^{2}(\tau)\right) \\
\cdot\left(1+13 h(\tau)+49 h^{2}(\tau)\right)^{1 / 3}
\end{gathered}
$$

Substituting (1.17) into the above equations, respectively, we obtain (1.8) and (1.9).

\section{The proofs of (1.10) and (1.11).}

Let

$$
f(z)=\frac{\theta_{1}(z \mid q) \theta_{1}\left(2 z \mid q^{7}\right)}{\theta_{1}^{11}\left(z \mid q^{7}\right)} .
$$

It is easy to check that $f(z)$ is an elliptic function with periods $\pi$ and $7 \pi \tau$. Also, $f(z)$ has only one pole at 0 , and its order is 9 . From lemma 2 we have

$$
\operatorname{res}(f ; 0)=0 \text {. }
$$

Set

$$
F(z):=z^{9} f(z), \quad \phi(z):=\frac{F^{\prime}(z)}{F(z)} .
$$

Using (2.10) we find that

$$
\begin{aligned}
\phi(z)= & \frac{9}{z}+\frac{\theta_{1}^{\prime}}{\theta_{1}}(z \mid q)-11 \frac{\theta_{1}^{\prime}}{\theta_{1}}\left(z \mid q^{7}\right)+2 \frac{\theta_{1}^{\prime}}{\theta_{1}}\left(2 z \mid q^{7}\right) \\
= & 2 z-\frac{2}{15} z^{3}-\frac{4}{35} z^{5}-\frac{246}{4725} z^{7}+4 \sum_{n=1}^{\infty} \frac{q^{n}}{1-q^{n}} \sin 2 n z \\
& +4 \sum_{n=1}^{\infty} \frac{q^{7 n}}{1-q^{7 n}}(2 \sin 4 n z-11 \sin 2 n z)+O\left(z^{9}\right) .
\end{aligned}
$$

It follows that

$$
\begin{aligned}
\phi^{\prime}(0) & =2 A(\tau) \\
\phi^{\prime \prime \prime}(0) & =-\frac{2}{15}(M(\tau)+5 M(7 \tau)), \\
\phi^{(5)}(0) & =-\frac{16}{63}(N(\tau)+53 N(7 \tau)), \\
\phi(0) & =\phi^{\prime \prime}(0)=\phi^{(4)}(0)=\phi^{(6)}(0)=0,
\end{aligned}
$$


and

(10.9) $\phi^{(7)}(0)=-\frac{16}{15}\left(1+480 \sum_{n=1}^{\infty} \frac{n^{7} q^{n}}{1-q^{n}}+245+245 \times 480 \frac{n^{7} q^{7 n}}{1-q^{7 n}}\right)$.

Employing the identity [1, p. 199], [19]

$$
M^{2}(\tau)=1+480 \sum_{n=1}^{\infty} \frac{n^{7} q^{n}}{1-q^{n}},
$$

Equation (10.9) can be written as

$$
\phi^{(7)}(0)=-\frac{16}{15}\left(M^{2}(\tau)+245 M^{2}(7 \tau)\right) .
$$

Using the fact that $\phi(0)=\phi^{\prime \prime}(0)=\phi^{(4)}(0)=\phi^{(6)}(0)=0$, we find that by a direct computation,

$$
\begin{aligned}
\operatorname{res}(f ; 0)=\frac{1}{8 !} & F(0)\left(105 \phi^{\prime}(0)^{4}+210 \phi^{\prime}(0)^{2} \phi^{\prime \prime \prime}(0)\right. \\
& \left.+28 \phi^{\prime}(0) \phi^{(5)}(0)+35 \phi^{\prime \prime \prime}(0)^{2}+\phi^{(7)}(0)\right) .
\end{aligned}
$$

Substituting (10.5), (10.6), (10.7), and (10.11) into (10.12) and then using (10.1) yields

$$
\begin{aligned}
& N(\tau)+53 N(7 \tau) \\
& =\frac{63}{8} A^{2}(\tau)(15 A(\tau)-M(\tau)-5 M(7 \tau)) \\
& \quad-\frac{1}{32 A(\tau)}\left(M^{2}(\tau)-14 M(\tau) M(7 \tau)+553 M^{2}(7 \tau)\right) .
\end{aligned}
$$

Replacing $\tau$ by $-1 / 7 \tau$ in the above equation and then applying (9.20)(9.23) in the resulting equation, we deduce that

$$
\begin{aligned}
53 N(\tau)+7^{6} N(7 \tau) \\
=-\frac{441}{8} A(\tau)\left(15 \times 7^{2} A^{2}(\tau)-5 M(\tau)-7^{4} M(7 \tau)\right) \\
\quad+\frac{1}{32 A(\tau)}\left(79 M^{2}(\tau)-2 \times 7^{4} M(\tau) M(7 \tau)+7^{7} M^{2}(7 \tau)\right) .
\end{aligned}
$$

Solving the above two equations for $N(\tau)$ and $N(7 \tau)$ we obtain the following lemma: 
Lemma 11. We have

$$
\begin{aligned}
N(\tau)= & \frac{49}{2320} A(\tau)\left(135 \times 7^{2} A^{2}(\tau)-2 \times 7^{4} M(7 \tau)-388 M(\tau)\right) \\
-\frac{1}{27840 A(\tau)}\left(7^{7} M^{2}(7 \tau)-6\right. & \times 7^{4} M(\tau) M(7 \tau) \\
& \left.+923 M^{2}(\tau)\right)
\end{aligned}
$$

(10.16) $\quad N(7 \tau)=\frac{7}{2320} A(\tau)\left(-135 A^{2}(\tau)+2 M(\tau)+388 M(\tau)\right)$

$$
\begin{aligned}
+\frac{1}{27840 A(\tau)}\left(M^{2}(\tau)-4\right. & 2 M(\tau) M(7 \tau) \\
+ & \left.6461 M^{2}(7 \tau)\right) .
\end{aligned}
$$

Substituting (1.8), (1.9), and (1.16) into the above two equations, respectively, we obtain (1.10) and (1.11).

\section{The proof of (1.18).}

In this section we first evaluate some elementary trigonometric sums. Let $\omega=\exp \left(\frac{2 \pi i}{7}\right)$. It is well-known that

$$
(1-x) \prod_{r=1}^{6}\left(1-x \omega^{r}\right)=1-x^{7} .
$$

It follows that for $x \neq 1$,

$$
\begin{aligned}
& \left(1-2 x \cos \frac{2 \pi}{7}+x^{2}\right)\left(1-2 x \cos \frac{4 \pi}{7}+x^{2}\right)\left(1-2 x \cos \frac{6 \pi}{7}+x^{2}\right) \\
& =\frac{1-x^{7}}{1-x} .
\end{aligned}
$$

Letting $x \rightarrow 1$ gives

$$
2^{6} \sin ^{2} \frac{\pi}{7} \sin ^{2} \frac{2 \pi}{7} \sin ^{2} \frac{3 \pi}{7}=7,
$$

and from this we obtain

$$
\sin \frac{\pi}{7} \sin \frac{2 \pi}{7} \sin \frac{3 \pi}{7}=\frac{1}{8} \sqrt{7}
$$

Similarly, setting $x=-1$ in (11.2), we have

$$
\cos \frac{\pi}{7} \cos \frac{2 \pi}{7} \cos \frac{3 \pi}{7}=\frac{1}{8} \text {. }
$$

Combining the above two equations we obtain

$$
\cot \frac{\pi}{7} \cot \frac{2 \pi}{7} \cot \frac{3 \pi}{7}=\frac{1}{\sqrt{7}} \text {. }
$$


We recall the identity (see (8.13))

$$
\cot \frac{\pi}{7}+\cot \frac{2 \pi}{7}-\cot \frac{3 \pi}{7}=\sqrt{7}
$$

Taking $q=0$ in (2.14), we obtain

$$
\cot ^{2} \frac{\pi}{7}+\cot ^{2} \frac{2 \pi}{7}+\cot ^{2} \frac{3 \pi}{7}=5 .
$$

From (11.6), (11.7), and (11.8), we readily find that

$$
\cot \frac{\pi}{7}, \quad \cot \frac{2 \pi}{7}, \quad \text { and } \quad-\cot \frac{3 \pi}{7}
$$

are the roots of cubic equation

$$
x^{3}-\sqrt{7} x^{2}+x+\frac{1}{\sqrt{7}}=0 .
$$

Let

$$
s_{n}=\cot ^{n} \frac{\pi}{7}+\cot ^{n} \frac{2 \pi}{7}+(-1)^{n} \cot ^{n} \frac{3 \pi}{7} .
$$

Then from (11.10) we obtain the following recurrence formula:

$$
s_{n+3}=\sqrt{7} s_{n+2}-s_{n+1}-\frac{1}{\sqrt{7}} s_{n}, \quad s_{0}=3, \quad s_{1}=\sqrt{7}, \quad s_{2}=5 .
$$

It follows that

$$
s_{3}=\frac{25}{\sqrt{7}}, \quad s_{4}=19, \quad s_{5}=\frac{103}{\sqrt{7}} .
$$

It can be easily verified that

$$
\cot ^{(4)} x=16 \cot x+40 \cot ^{3} x+24 \cot ^{5} x .
$$

Therefore we have

$$
\cot ^{(4)} \frac{\pi}{7}+\cot ^{(4)} \frac{2 \pi}{7}+\cot ^{(4)} \frac{3 \pi}{7}=16 s_{1}+40 s_{3}+24 s_{5}=\frac{3584}{\sqrt{7}} .
$$

Now we begin to prove (1.18). Using (2.4) we can verify that

$$
f(z)=\frac{\theta_{1}(2 z \mid q) \theta_{1}\left(z+\frac{\pi}{7} \mid q\right) \theta_{1}\left(z+\frac{2 \pi}{7} \mid q\right) \theta_{1}\left(z-\frac{3 \pi}{7} \mid q\right)}{\theta_{1}^{7}(z \mid q)}
$$

is an elliptic function with only one pole, namely, at 0 with order 6 .

Set

$$
F(z):=z^{6} f(z), \quad \phi(z):=\frac{F^{\prime}(z)}{F(z)}
$$


We find that

$$
\begin{aligned}
\phi(z)= & \frac{6}{z}-7 \frac{\theta_{1}^{\prime}}{\theta_{1}}(z \mid q)+2 \frac{\theta_{1}^{\prime}}{\theta_{1}}(z \mid q) \\
& +\frac{\theta_{1}^{\prime}}{\theta_{1}}\left(z+\frac{\pi}{7} \mid q\right)+\frac{\theta_{1}^{\prime}}{\theta_{1}}\left(z+\frac{2 \pi}{7} \mid q\right)+\frac{\theta_{1}^{\prime}}{\theta_{1}}\left(z-\frac{3 \pi}{7} \mid q\right) \\
= & L(\tau) z-\frac{z^{3}}{5} M(\tau)+\frac{\theta_{1}^{\prime}}{\theta_{1}}\left(z+\frac{\pi}{7} \mid q\right) \\
& +\frac{\theta_{1}^{\prime}}{\theta_{1}}\left(z+\frac{2 \pi}{7} \mid q\right)+\frac{\theta_{1}^{\prime}}{\theta_{1}}\left(z-\frac{3 \pi}{7} \mid q\right)+O\left(z^{5}\right) .
\end{aligned}
$$

Setting $z=0$ and then using (8.19), we find that

$$
\begin{aligned}
\phi(0) & =\frac{\theta_{1}^{\prime}}{\theta_{1}}\left(\frac{\pi}{7} \mid q\right)+\frac{\theta_{1}^{\prime}}{\theta_{1}}\left(\frac{2 \pi}{7} \mid q\right)-\frac{\theta_{1}^{\prime}}{\theta_{1}}\left(\frac{3 \pi}{7} \mid q\right) \\
& =\sqrt{7}\left(1+2 \sum_{n=1}^{\infty}\left(\frac{n}{7}\right) \frac{q^{n}}{1-q^{n}}\right) .
\end{aligned}
$$

Differentiating (11.18) with repect to $z$ and then setting $z=0$ and finally using (2.14), we obtain

$$
\begin{aligned}
(11.20) \phi^{\prime}(0) & =L(\tau)+\left(\frac{\theta_{1}^{\prime}}{\theta_{1}}\right)^{\prime}\left(\frac{\pi}{7} \mid q\right)+\left(\frac{\theta_{1}^{\prime}}{\theta_{1}}\right)^{\prime}\left(\frac{2 \pi}{7} \mid q\right)+\left(\frac{\theta_{1}^{\prime}}{\theta_{1}}\right)^{\prime}\left(\frac{3 \pi}{7} \mid q\right) \\
& =-7 A(\tau) .
\end{aligned}
$$

Differentiating (11.18) twice with repect to $z$, seting $z=0$, and using (8.6) and (8.7), we obtain

$$
\begin{aligned}
\phi^{\prime \prime}(0) & =\left(\frac{\theta_{1}^{\prime}}{\theta_{1}}\right)^{\prime \prime}\left(\frac{\pi}{7} \mid q\right)+\left(\frac{\theta_{1}^{\prime}}{\theta_{1}}\right)^{\prime \prime}\left(\frac{2 \pi}{7} \mid q\right)-\left(\frac{\theta_{1}^{\prime}}{\theta_{1}}\right)^{\prime \prime}\left(\frac{3 \pi}{7} \mid q\right) \\
& =\frac{8}{\sqrt{7}}\left(8-7 \sum_{n=1}^{\infty}\left(\frac{n}{7}\right) \frac{n^{2} q^{n}}{1-q^{n}}\right) .
\end{aligned}
$$

Using (2.15), we find that

$$
\begin{aligned}
\phi^{\prime \prime \prime}(0)= & -\frac{6}{5} M(\tau)+\left(\frac{\theta_{1}^{\prime}}{\theta_{1}}\right)^{\prime \prime \prime}\left(\frac{\pi}{7} \mid q\right) \\
& +\left(\frac{\theta_{1}^{\prime}}{\theta_{1}}\right)^{\prime \prime \prime}\left(\frac{2 \pi}{7} \mid q\right)+\left(\frac{\theta_{1}^{\prime}}{\theta_{1}}\right)^{\prime \prime \prime}\left(\frac{3 \pi}{7} \mid q\right) \\
= & -\frac{1}{15}(7 M(\tau)+2401 M(7 \tau)) .
\end{aligned}
$$


From (11.16) and (8.7), we have

$$
\begin{aligned}
\phi^{(4)}(0)= & \left(\frac{\theta_{1}^{\prime}}{\theta_{1}}\right)^{(4)}\left(\frac{\pi}{7} \mid q\right)+\left(\frac{\theta_{1}^{\prime}}{\theta_{1}}\right)^{(4)}\left(\frac{2 \pi}{7} \mid q\right)-\left(\frac{\theta_{1}^{\prime}}{\theta_{1}}\right)^{(4)}\left(\frac{3 \pi}{7} \mid q\right) \\
= & \cot ^{(4)} \frac{\pi}{7}+\cot ^{(4)} \frac{2 \pi}{7}+\cot ^{(4)} \frac{3 \pi}{7} \\
& +64 \sum_{n=1}^{\infty} \frac{n^{4} q^{n}}{1-q^{n}}\left(\sin \frac{2 n \pi}{7}+\sin \frac{4 n \pi}{7}-\sin \frac{6 n \pi}{7}\right) \\
= & 32 \sqrt{7}\left(16+\sum_{n=1}^{\infty}\left(\frac{n}{7}\right) \frac{n^{4} q^{n}}{1-q^{n}}\right) .
\end{aligned}
$$

By logarithmic differentiation we find that

$$
\begin{aligned}
\operatorname{res}(f ; 0)= & \frac{1}{120} F(0)\left(\phi(0)^{5}+10 \phi(0)^{3} \phi^{\prime}(0)+5 \phi(0) \phi^{\prime \prime \prime}(0)\right. \\
& +10 \phi(0)^{2} \phi^{\prime \prime}(0)+15 \phi(0) \phi^{\prime}(0)^{2} \\
& \left.+10 \phi^{\prime}(0) \phi^{\prime \prime}(0)+\phi^{(4)}(0)\right)
\end{aligned}
$$

Substituting (11.19)-(11.23) into the above equation and then using (8.19) in the resulting equation and finally using the fact that $\operatorname{res}(f ; 0)=0$, we obtain

$$
\begin{aligned}
96 & \left(16+\sum_{n=1}^{\infty}\left(\frac{n}{7}\right) \frac{n^{4} q^{n}}{1-q^{n}}\right) \\
= & \left(1+2 \sum_{n=1}^{\infty}\left(\frac{n}{7}\right) \frac{q^{n}}{1-q^{n}}\right)(17 M(\tau)+2401 M(7 \tau)) \\
& -882\left(1+2 \sum_{n=1}^{\infty}\left(\frac{n}{7}\right) \frac{q^{n}}{1-q^{n}}\right)^{5}
\end{aligned}
$$

Substituting (1.8), (1.9), and (1.16) into the above equation we immediately obtain (1.18).

Acknowledgements. This work is supported, in part, by Academic Research Fund R146000027112 from the National University of Singapore. The author is grateful to the referee for his many helpful suggestions leading to an improvement of an earlier version of this paper. He would also like to thank Heng Huat Chan, Li-Chien Shen, and Yifan Yang for their comments. 


\section{References}

[1] T.M. Apostol, Modular Functions and Dirichlet Series in Number Theory, SpringerVerlag, New York, 1976, MR 54 \#10149, Zbl 0332.10017.

[2] B.C. Berndt, Ramanujan's Notebooks, Part III, Springer-Verlag, New York, 1991, MR 92j:01069, Zbl 0733.11001.

[3] B.C. Berndt, H.H. Chan, J. Sohn and S.H. Son, Eisenstein series in Ramanujan's lost notebook, Ramanujan J., 4 (2000), 81-114, MR 2001j:11018.

[4] B.C. Berndt and L.-C. Zhang, A new class of theta-function identities originating in Ramanujan's notebooks, J. Number Theory, 48 (1994), 224-242, MR 95m:11046, Zbl 0812.11028.

[5] K. Chandrasekharan, Elliptic Functions, Springer-Verlag, New York, 1985, MR 87e:11058, Zbl 0575.33001.

[6] R.J. Evans, Theta function identities, J. Math. Anal. Appl., 147 (1990), 97-121, MR 91d:11045, Zbl 0707.11033.

[7] F.G. Garvan, A simple proof of Watson's partition congruences for powers of 7, J. Austral. Math. Soc. (Series A), 36 (1984), 316-334, MR 85f:11072, Zbl 0533.10006.

[8] M.D. Hirschhorn and D.C. Hunt, A simple proof of the Ramanujan conjecture for powers of 5, J. Reine Angew. Math., 326 (1981), 1-17, MR 82m:10023, Zbl 0452.10015.

[9] Z.-G. Liu, The Borweins' cubic theta function identity and some cubic modular identities of Ramanujan, Ramanujan J., 4 (2000), 43-50, MR 2001f:33026.

[10] _ On certain identities of Ramanujan, J. Number Theory, 83 (2000), 59-75, MR 2001f:11066, Zbl 0981.11016.

[11] _ Some Eisenstein series identities, J. Number Theory, 85 (2000), 231-252, MR 2001k:11075, Zbl 0973.11053.

[12] Some theta function identities associated with the modular equations of degree 5, Integers: Electronic Journal of Combinatorial Number Theory, 1 (2001), A\#03, 14pp., MR 2002c:33017.

[13] _ Residue theorem and theta function identities, Ramanujan J., 5 (2001), 129151, MR 2002g:11056.

[14] S. McCullough and L.-C. Shen, On the Szgö kernel of an annulus, Proc. Amer. Math. Soc., 121 (1994), 1110-1120, MR 94j:30005, Zbl 0807.30005.

[15] S. Raghavan, On certain identities due to Ramanujan, Quart. J. Math. Oxford(2), 37 (1986), 221-229, MR 87j:11113, Zbl 0585.10033.

[16] S. Raghavan and S.S. Rangachari, Ramanujan's elliptic integrals and modular identities, in 'Number Theory and Related Topics', Oxford University Press, Bombay, 1989, 119-149, MR 98b:11045, Zbl 0748.33013.

[17] S. Ramanujan, The Lost Notebook and Other Unpublished Papers, Narosa, New Delhi, 1988, MR 89j:01078, Zbl 0639.01023.

[18] _ Notebooks (2 volumes), Tata Institute of Fundamental Research, Bombay, 1957, MR 20 \#6340, Zbl 0138.24201.

[19] _ On certain arithmetical functions, Trans. Cambridge Philos. Soc., 22 (1916), 159-184.

[20] L.-C. Shen, On the additive formula of the theta functions and a collection of Lambert series pertaining to the modular equations of degree 5, Trans. Amer. Math. Soc., 345 (1994), 323-345, MR 95a:33038, Zbl 0808.33014. 
[21] _ On the products of three theta functions, Ramanujan J., 3 (1999), 343-357, MR 2000k:33038.

[22] G.N. Watson, Ramanujan Vermutung üeber Zerfällungsanzahlen, J. Reine Angew. Math., 179 (1938), 97-128.

[23] E.T. Whittaker and G.N. Watson, A Course of Modern Analysis, 4th ed. Cambridge Univ. Press, Cambridge, 1966, MR 97k:01072, Zbl 0951.30002.

Received August 28, 2001 and revised October 22, 2001.

Department of Mathematics

East China Normal University

SHANGHAI 200062

P. R. of China

E-mail address: liuzg18@hotmail.com 